\title{
Upwelling Variability Offshore of Dakhla, Southern Morocco
}

By Ahmed Makaoui, Younes Belabchir, Ismail Bessa, Abedelaziz Agouzouk, Mohammed Idrissi, Omar Ettahiri, and Karim Hilmi

To enhance oceanographic observation capacities in the Atlantic Ocean and the Mediterranean Sea, and under the project "Observation of the Marine Environment with an Operational Oceanographic Observation System," Morocco's National Institute of Fisheries Research (INRH) acquired and installed its first meteorological and oceanographic buoy offshore of Dakhla, southern Morocco (Figure 1). This buoy, moored at a fixed point $\left(23^{\circ} 55.1208^{\prime} \mathrm{N}, 1^{\circ} 11.1569^{\prime} \mathrm{W}\right)$ on October 7, 2016, is a first experiment conducted by Moroccan scientists and INRH along the Moroccan Atlantic coast. The project's intent was to allow continuous, real-time acquisition of meteorological and oceanographic data from the South Morocco upwelling area, which is of great scientific, oceanographic, and environmental utility for maritime decision-making, in particular, for the marine fisheries and aquaculture sector in a context of climate change.

For surface measurements, the buoy carries meteorological instruments, including an anemometer (wind speed and direction) and air temperature and atmospheric pressure sensors. Beneath the surface, the buoy is equipped with a sensor for measuring the wave swell (height, direction, and period); a profiler for measuring the current (speed and direction) in the water column; a multiparameter sensor that includes water temperature (range $\left.0^{\circ}-40^{\circ} \mathrm{C}\right)$, salinity $(0-40 \mathrm{psu})$, fluorescence, and dissolved oxygen at a single depth level. In addition, the buoy is outfitted with a GPS system for continuous positioning and a complete data acquisition and transmission system.

When launched, the buoy recorded and transmitted data to the Laboratory of Physics and Marine Biogeochemistry of the INRH Regional Center in Casablanca. Data processing allowed monitoring of the different parameters every 30 minutes. The results recorded during the month of November 2016 detected the beginning of the upwelling process that brings deep, cold, and nutrient-rich waters to the surface, increasing biological activity and intensifying the current (Figure 2). Surface water temperatures cooled from $22.5^{\circ} \mathrm{C}$ to $18.5^{\circ} \mathrm{C}$ on November 10 , predicating upwelling during the rest of the month. Salinity followed the same evolution, decreasing from 38.5 psu to $35.6 \mathrm{psu}$, then stabilizing between 36.5 psu and 37.5 psu. The profile of dissolved oxygen concentration indicates good oxygenation in the area, varying between $8 \mathrm{mg} / \mathrm{L}$ and $9 \mathrm{mg} / \mathrm{L}$ until upwelling begins, when it starts to decrease below $6 \mathrm{mg} / \mathrm{L}$ for three days before returning to concentrations above $7 \mathrm{mg} / \mathrm{L}$ for the rest of the month. These conditions were accompanied by phytoplankton enrichment manifested by an increase in the concentration of chlorophyll-a from $0.08 \mu \mathrm{g} / \mathrm{L}$ to close to $0.12 \mu \mathrm{g} / \mathrm{L}$. The $\mathrm{pH}$ was stable at 8
FIGURE 1. This moored buoy was installed on October 7, 2016, offshore Dakhla, southern Morocco to collect meteorological and oceanographic data. It has been out of commission since early March 2017 due to vandalism.
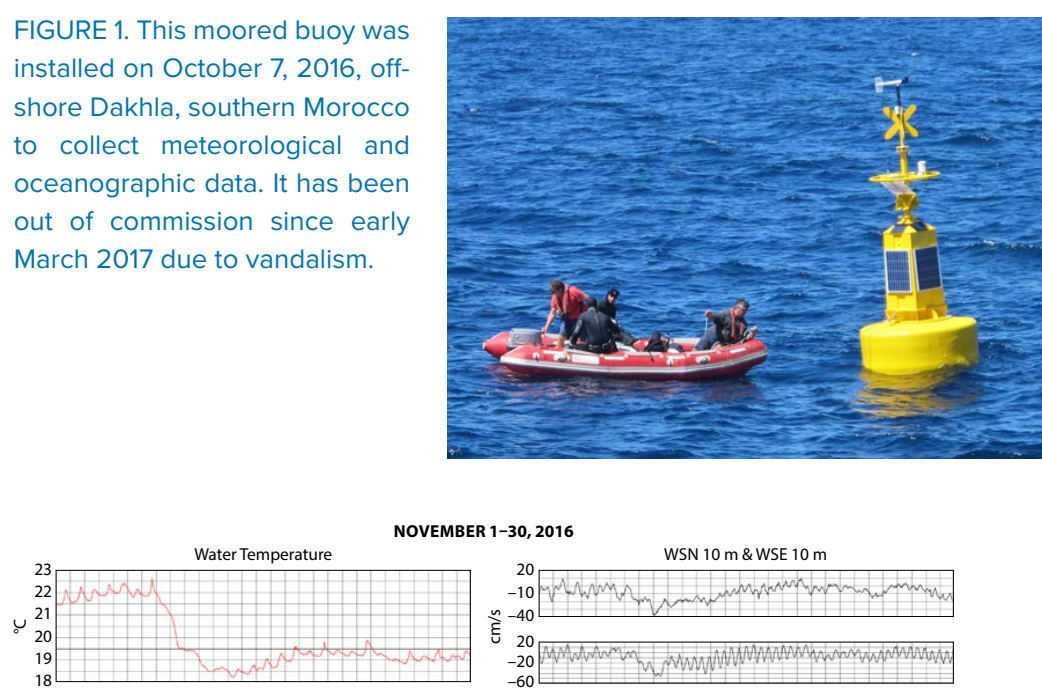

Salinity
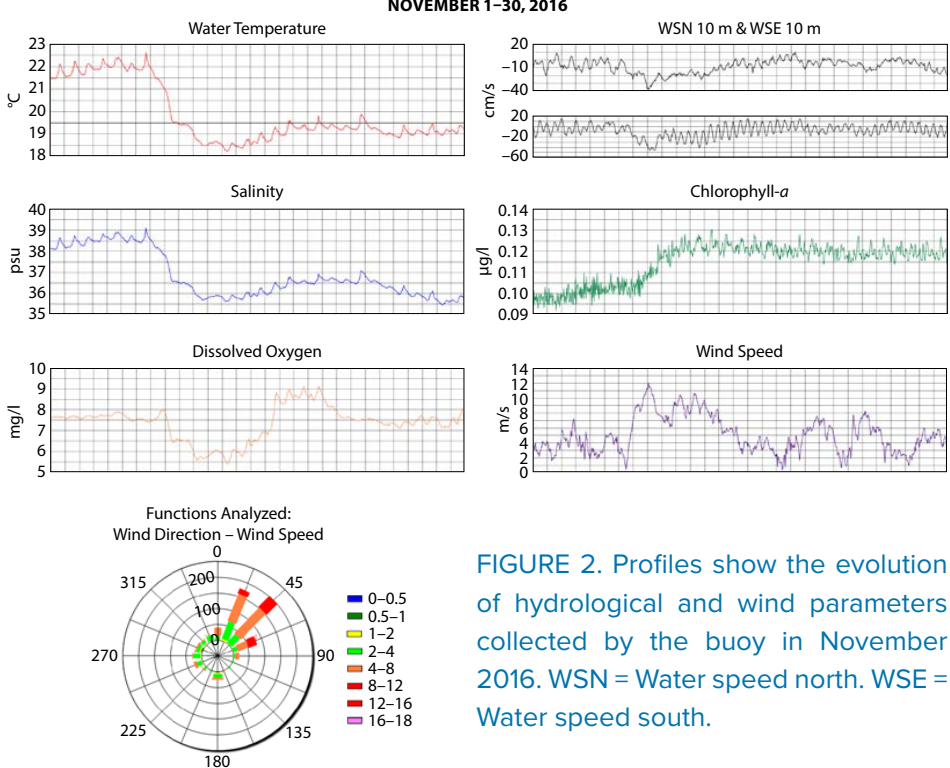

Chlorophyll-a

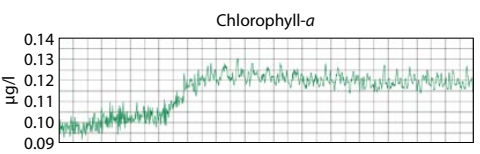

Wind Speed

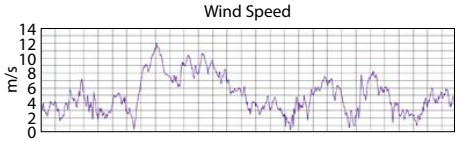

FIGURE 2. Profiles show the evolution of hydrological and wind parameters collected by the buoy in November 2016. $\mathrm{WSN}=$ Water speed north. WSE = Water speed south.

during November. The south to southwest marine current recorded at $10 \mathrm{~m}$ depth increased during the period of upwelling activity and reached $35 \mathrm{~cm} / \mathrm{s}$ for the southern component and more than $40 \mathrm{~cm} / \mathrm{s}$ for its western component. Maximum intensity was recorded on November 9 just before upwelling began.

While vandalism five months after buoy deployment has delayed this project coming to fruition, these early data demonstrate that the selected site for the buoy was optimal, recording an important correlation between hydrological and meteorological parameters, especially the change in wind strength and direction, which activates upwelling. It is a constant challenge here, and in other areas of intense fishing activity, to maintain fixed observing assets and keep them safe from vandalism and accidental damage. We are optimistic that when repairs to the buoy are complete and it is redeployed, we will be able to meet our objectives.

ARTICLE DOI: https://doi.org/10.5670/oceanog.2021.supplement.02-20 


\section{AUTHORS}

Ahmed Makaoui, Younes Belabchir, Ismail Bessa, Abedelaziz Agouzouk, Mohammed Idrissi (idrissi@inrh.ma), Omar Ettahiri, and Karim Hilmi, Laboratoire de Physique et Bio-géochimie Marine, Institut National de Recherche Halieutique (INRH), Morocco.

\section{ARTICLE CITATION}

Makaoui, A., Y. Belabchir, I. Bessa, A. Agouzouk, M. Idrissi, O. Ettahiri, and K. Hilmi. 2021. Upwelling variability offshore of Dakhla, southern Morocco. P. 49 in Frontiers in Ocean Observing: Documenting Ecosystems, Understanding Environmental Changes, Forecasting Hazards. E.S. Kappel, S.K. Juniper, S. Seeyave, E. Smith, and M. Visbeck, eds, A Supplement to Oceanography 34(4), https://doi.org/10.5670/oceanog.2021. supplement.02-20.

\section{COPYRIGHT \& USAGE}

This is an open access article made available under the terms of the Creative Commons Attribution 4.0 International License (https://creativecommons.org/ licenses/by/4.0/), which permits use, sharing, adaptation, distribution, and reproduction in any medium or format as long as users cite the materials appropriately, provide a link to the Creative Commons license, and indicate the changes that were made to the original content. 